\title{
Investigation of Plasmon Resonance of Stacked Gold Nanodisks using Time-Domain Simulations
}

\author{
${ }^{1}$ D. Baumann, ${ }^{2}$ R. Giannini, ${ }^{1}$ C. Hafner, ${ }^{3}$ E.P. Li, ${ }^{4}$ C. Fumeaux \\ ${ }^{1}$ Laboratory for Electromagnetic Fields and Microwave Electronics (IFH), ETH Zurich, Switzerland \\ ${ }^{2}$ Laboratory of Metal Physics and Technology (LMPT), ETH Zurich, Switzerland \\ ${ }^{3}$ Institute of High Performance Computing (IHPC), A*STAR, Singapore \\ ${ }^{4}$ School of Electrical \& Electronic Engineering, The University of Adelaide, Australia
}

\begin{abstract}
Plasmon resonances are able to produce a large field enhancement, which can be exploited for various purposes, such as biosensing or optical mixing. In this paper, the plasmon resonance of two stacked gold nanodisks separated by a tiny $2 \mathrm{~nm}$ gap is studied in time-domain simulations, offering the possibility of transient and broadband analysis. Moreover, time-domain simulations are amenable to extensions towards nonlinear models. For efficient simulations, the extremely small gap in between the nanodisks, as well as potential fabrication imperfections, are advantageously modeled with an unstructured mesh. This paper presents 3D simulations of the nanodisk plasmon problem using the finite-volume time-domain method in a tetrahedral mesh.
\end{abstract}

\section{INTRODUCTION}

Electromagnetic waves at visible frequencies incident on a metallic-dielectric interface are able to produce plasmon resonances. These collective oscillations of electrons can be exploited for various purposes, ranging from biosensing, over optical mixing to nano antennas and sensing of single molecules [1-4]. To support the progress in nano-fabrication and $3 \mathrm{D}$ patterning of metals at nanometer scale, the numerical simulation of plasmonic structures have become indispensible to model plasmonic phenomena. Such simulations are however very demanding and suitable electromagnetic field solvers have to be indentified.

In this paper, the surface plasmon resonance in the gap between two stacked gold nanodisks is studied using broadband time-domain simulations. In the present problem, the gold disks [3] are placed extremely close to each other, which is very demanding for 3D simulations. To adequately resolve the tiny gap, the necessary small cell size can lead to an uncomfortable increase of the number of elements in 3D if a structured mesh is used. In contrast, an unstructured inhomogeneous mesh can alleviate this problem and may further be more appropriate to include other effects, such as geometric imperfections due to the fabrication process, which might result in non-canonical shapes, irregular edges, or rough surfaces, etc. [5]. The Finite-Volume Time-Domain (FVTD) method is chosen here as an adequate and versatile numerical tool for this kind of simulations [6]. The application in time domain (TD) makes the method compliant to the incorporation of non-linear effects [7]. Additionally, FVTD exhibits two main advantages. Firstly, FVTD is based on an explicit timestepping scheme and secondly, FVTD is applied in a tetrahedral, unstructured and potentially strongly inhomogeneous mesh. For efficient simulations the inhomogeneity of the mesh is combined with a local-time stepping scheme [8].

\section{PROBLEM DESCRIPTION}

In order to achieve a strong plasmon resonance, two gold disks are stacked above each other, leaving a small gap in between them. Depending on the size of the gap and the material inside the gap, a strong field enhancement is expected. In the following, the gap distance between the two stacked gold nanodisks is chosen as $d=2 \mathrm{~nm}$ and the disks have a radius of $r=70 \mathrm{~nm}$ and a height of $h=60 \mathrm{~nm}$. This geometry configuration is depicted in Fig. 1. The disks are illuminated perpendicularly to the $z$-axis by a plane wave covering the wavelength spectrum from $600 \mathrm{~nm}$ to $1600 \mathrm{~nm}$.

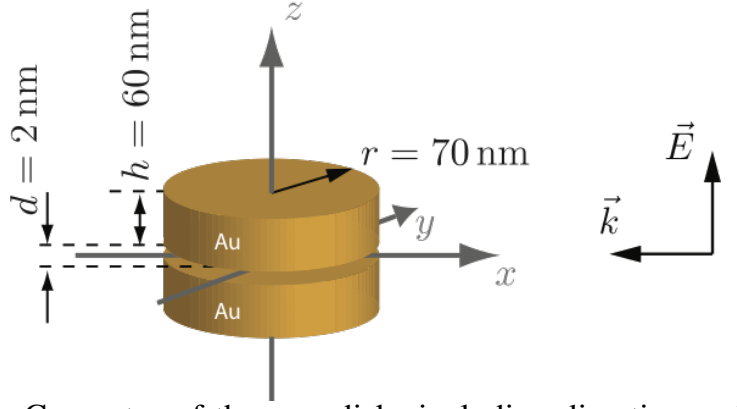

Fig. 1: Geometry of the nanodisks including direction and polarization of the impinging electromagnetic wave.

The field enhancement observable in two different nanodisk configurations is investigated in this paper: 1) a canonical investigation of two stacked disks where the gap in between is filled with air, and 2) a more realistic approach, where the gap is filled with silicon dioxide $\left(\mathrm{SiO}_{2}, \varepsilon_{\mathrm{r}}=2.31\right)$.

\section{GOLD NANO-DISK SIMULATION}

The gold disk configuration is simulated using two different numerical methods, the first one applied in frequency domain (FD) and the second applied in time domain (TD). For the FD simulation, the commercially available JCMsuite software [9] is employed, which is based on the finite-element method. The TD simulations are performed using the FVTD method.

\subsection{JCMsuite}

For axis-symmetric problems, JCMsuite offers a decomposition of the electromagnetic field in a Fourier series expressing the azimuthal dependence of the fields. With this approach, no gridding in azimuthal direction is necessary, reducing this approach to a $2 \mathrm{D}$ representation of the $3 \mathrm{D}$ problem. It is important to note that only the geometry has to be axissymmetric. The excitation, as shown in Fig. 1, does not have to exhibit the same symmetry. Since only a $2 \mathrm{D}$ problem has to be 
solved numerically, the expected computational costs are comparably low, however, since applied in FD, the simulation has to be repeated for all frequency points of interest. Nevertheless, if necessary, JCMsuite is also capable of solving a full 3D problem. In this paper, the simulation results of JCMsuite are used as a reference for validating the FVTD method results.

\subsection{FVTD}

For possibly non-symmetric structures, such as two gold disks placed next to each other on a substrate, or to include fabrication imperfections such as non-cylindrical shapes, rough surfaces or irregular edges, a full 3D simulation has to be performed. The nature of the unstructured and inhomogeneous tetrahedral mesh employed in the FVTD method allows the resolution of fine structural details in an electrically large system [10]. In the present case, this is very convenient to resolve the gap region in between the two nanodisks. The triangular surface mesh of the FVTD model is shown in Fig. 2, illustrating the strong mesh inhomogeneity, which is required to resolve the very thin gap region. In addition, it can be emphasized that the gold nanodisks require a relatively fine discretization compared to the surrounding medium (free space).
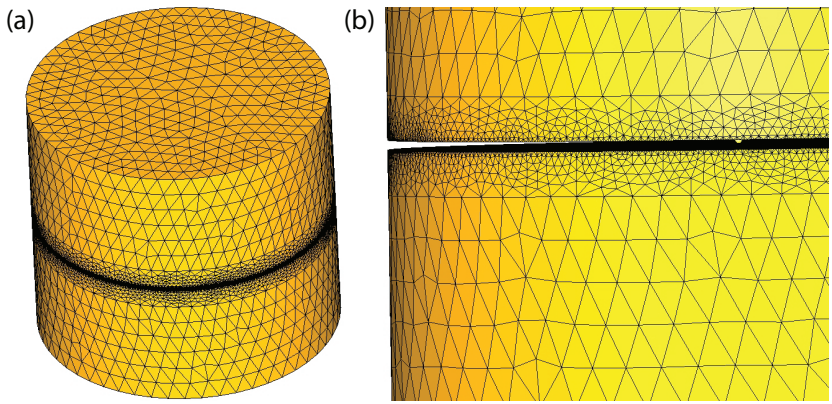

Fig. 2: (a) Surface mesh of the FVTD model, (b) close-up view of the gap region. The tetrahedral volume mesh is not shown for the sake of a better visibility.

The strong inhomogeneity of the mesh prevents a 3D explosion of number of cells, and moreover redounds to FVTD's advantage by applying a geometry-matched local time-stepping (LTS) scheme. The LTS typically yields computational speedup factors around of 6 to 10, depending on the problem [8]. For the present FVTD simulation, it is sufficient to model only a quarter of the structure, because of the axis symmetry of the stacked nanodisks. As indicated in Fig. 3, (a) a perfectly electric conducting (PEC), and (b) a perfectly magnetic conducting (PMC) symmetry plane can be exploited, reducing the degrees of freedom of the simulation by a factor of four.

In TD, the whole frequency band of interest can be simulated in one simulation run using an appropriate excitation pulse, as shown in Fig. 4 (a). However, the system response of the highly resonant structure of the stacked nanodisks exhibits an extremely extended ringing, making long simulation times necessary, which becomes apparent in Fig. 4 (b), where the time evolution of the electric field in the center of the gap is plotted. Only after around $300 \mathrm{fs}$, which is 50 times the duration of the excitation pulse, the electromagnetic field in the tiny gap has decayed sufficiently to reflect accurately the steady state (in FD) after Fourier analysis.
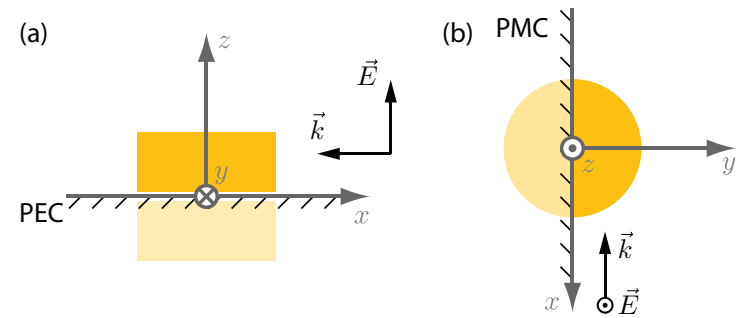

Fig. 3: (a) PEC and (b) PMC symmetry planes exploited in the FVTD simulation.

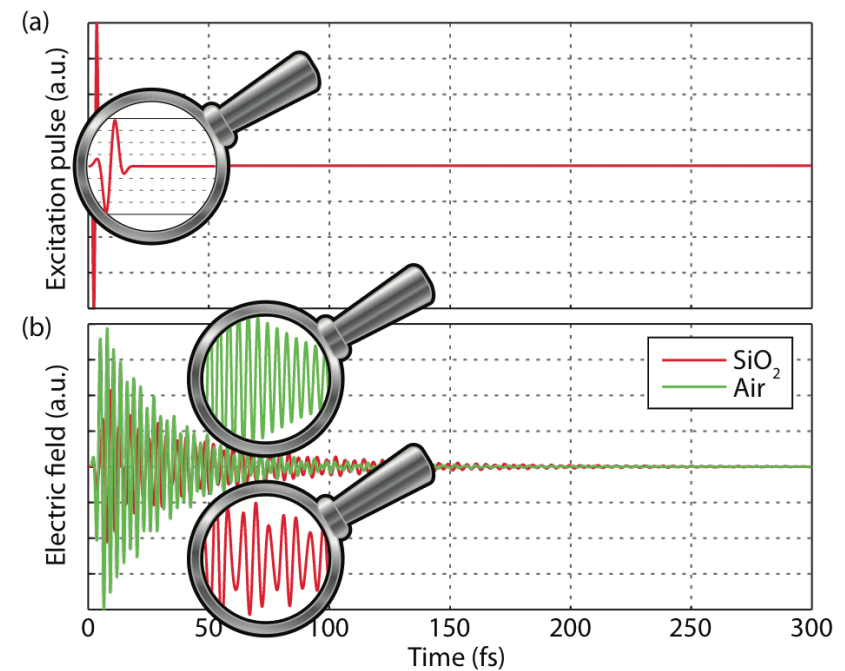

Fig. 4: (a) Gaussian pulse used as excitation, (b) system response at the center of the structure in the gap filled with air (green line) and $\mathrm{SiO}_{2}$ (red line).

The magnifying glasses in Fig. 4 (b) provide a closer look at the time-dependent behavior of the electric field in the center of the gap. A single frequency sinusoid is observed in the airfilled gap, and a beat frequency in the $\mathrm{SiO}_{2}$-filled gap, both with an exponentially decaying envelope. This suggests that in the observed frequency range a single resonance exists in the center of the air-filled gap. In contrast, there are multiple resonances observable in the $\mathrm{SiO}_{2}$-filled gap, resulting in the beat behavior. This is confirmed in Fig. 5, depicting the FD characteristics of the electric field in the center of the gap. The $\mathrm{SiO}_{2}$-filled gap has several distinct resonances, with the most prominent at $850 \mathrm{~nm}$ and $1165 \mathrm{~nm}$, whereas the electric field in the center of the air-filled gap shows a single resonance at $830 \mathrm{~nm}$. The evolution of these resonances as a function of the location in the gap will be discussed in more detail in section 5 .

Unfortunately, for the modeling of dispersive materials in TD simulations, it is not straightforward to use measured FD material parameters. An adequate fitting to analytical material 
models such as Debye, Lorentz and Drude models has to be performed. How this is done in the FVTD method, is explained in the following section.

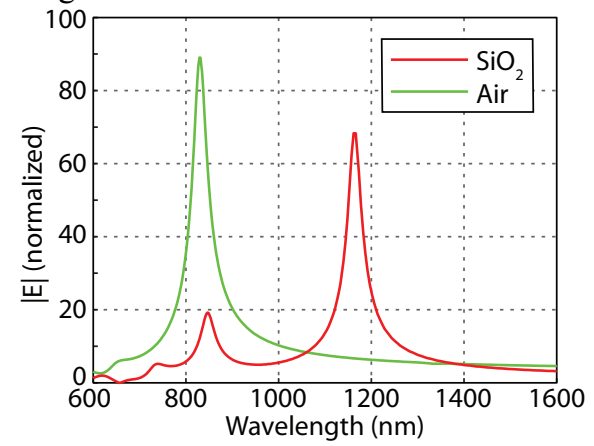

Fig. 5: FVTD simulation of field enhancement in the gap filled with air and $\mathrm{SiO}_{2}$ in the center of the gap at $0 \mathrm{~nm}$.

\begin{tabular}{|l|l|l|}
\hline$\varepsilon_{\infty}$ & 3.65 & $\mathrm{n}=2$ \\
\hline Lorentz & $\mathrm{n}=1$ & 1.89 \\
\hline \multirow{2}{*}{$\begin{array}{l}\Delta \varepsilon_{n}^{\mathrm{Lo}} \\
\omega_{n}^{\mathrm{Lo}} \\
\gamma_{n}^{\mathrm{Lo}}\end{array}$} & 1.47 & $6.45 \cdot 10^{15} \mathrm{~s}^{-1}$ \\
\cline { 2 - 2 } & $4.79 \cdot 10^{15} \mathrm{~s}^{-1}$ & $1.39 \cdot 10^{15} \mathrm{~s}^{-1}$ \\
\cline { 2 - 2 } Drude & $9.08 \cdot 10^{14} \mathrm{~s}^{-1}$ & \\
\hline$\omega_{p}^{\text {Dr }}$ & $\mathrm{p}=1$ & \multicolumn{1}{|}{} \\
$\gamma_{p}^{\text {Dr }}$ & $1.28 \cdot 10^{16} \mathrm{~s}^{-1}$ & \\
\cline { 2 - 3 } & $2.76 \cdot 10^{13} \mathrm{~s}^{-1}$ & \\
\hline
\end{tabular}

Table 1: Parameters of the Lorentz-Drude model to fit the measured permittivity of gold in the range of 300 to $1600 \mathrm{~nm}$.

\section{DISPERSIVE MATERIAL DESCRIPTION}

Since the finite-volume technique is applied in time domain, the material characteristic of gold has to be approximated by an appropriate material model. In FVTD, a modular usage of dispersive material models is implemented, allowing a flexible handling of a mixed $P$-pole Debye (superscript De), Lorentz (Lo) and Drude (Dr) models

$$
\begin{aligned}
\varepsilon_{r}=\varepsilon_{\infty} & +\sum_{m} \frac{\Delta \varepsilon_{m}^{\mathrm{De}}}{1+j \omega \gamma_{m}^{D e}} \\
& +\sum_{n} \frac{\Delta \varepsilon_{n}^{\mathrm{Lo}} \cdot\left(\omega_{n}^{\mathrm{Lo}}\right)^{2}}{\left(\omega_{n}^{\mathrm{L} o}\right)^{2}+2 j \omega \gamma_{n}^{\mathrm{Lo}}-\omega^{2}}-\sum_{p} \frac{\left(\omega_{p}^{\mathrm{Dr}}\right)^{2}}{\omega^{2}-j \omega \gamma_{p}^{\mathrm{Dr}}} .
\end{aligned}
$$

For the representation of gold at visible frequencies, a double Lorentz, single Drude model was found to be suitable

$$
\varepsilon_{r}=\varepsilon_{\infty}+\sum_{n=1}^{2} \frac{\Delta \varepsilon_{n}^{\mathrm{Lo}} \cdot\left(\omega_{n}^{\mathrm{Lo}}\right)^{2}}{\left(\omega_{n}^{\mathrm{Lo}}\right)^{2}+2 j \omega \gamma_{n}^{\mathrm{Lo}}-\omega^{2}}-\frac{\left(\omega_{1}^{\mathrm{Dr}}\right)^{2}}{\omega^{2}-j \omega \gamma_{1}^{\mathrm{Dr}}}
$$

with material parameters listed in Table 1 [11]. The material model (2) accurately represents the measured permittivity of gold in a wide frequency range. Fig. 6 compares the measured values of the gold permittivity (dashed lines) to corresponding values obtained with the fitting parameters (solid lines). A reasonable agreement is achieved for both, real and imaginary part of $\varepsilon_{\mathrm{r}}$, over the wavelength band from $300 \mathrm{~nm}$ to $1600 \mathrm{~nm}$.
Since the behavior of the permittivity of gold is not smooth, especially in between $300 \mathrm{~nm}$ and $600 \mathrm{~nm}$, its modeling with analytical functions as (1) is a challenging task and three poles are required to find a satisfying approximation.

In order to enable a fair comparison between the two different computational algorithms, the analytical gold permittivity values based on a two-pole Lorentz, one-pole Drude model (2) are employed in both, JCMsuite and FVTD simulations, in the following.

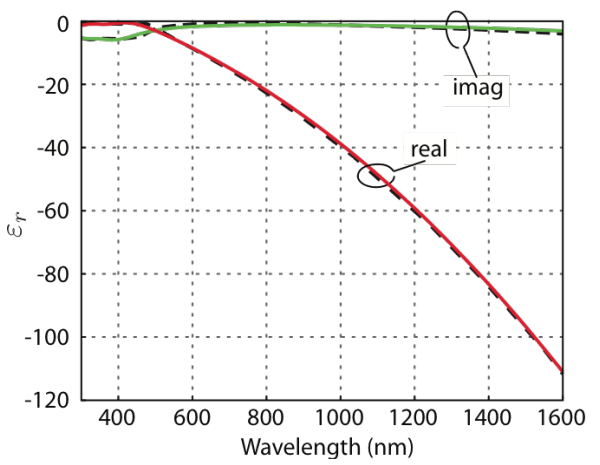

Fig. 6: Fitted (solid lines) versus measured (dashed lines) bulk gold permittivity.

\section{RESULTS}

In the following, the discussed quantity of interest is the field enhancement, which is defined as the absolute value of the FD electric field, normalized to the FD excitation amplitude. Fig. 7 depicts the field enhancement at different positions in the $2 \mathrm{~nm}$ wide gap region as a function of wavelength. According to the coordinate system indicated in Fig. 1, the electric field is plotted at four locations on the $x$-axis, namely at $x=5 \mathrm{~nm}, x=30 \mathrm{~nm}, x=50 \mathrm{~nm}$, and $x=65 \mathrm{~nm}$. Resonances can be observed at $830 \mathrm{~nm}$ and $1335 \mathrm{~nm}$ in the case of an airfilled gap and at $850 \mathrm{~nm}, 965 \mathrm{~nm}$ and $1165 \mathrm{~nm}$ in the case of a $\mathrm{SiO}_{2}$-filled gap. For both cases, the FVTD solution (solid lines) is compared to the JCMsuite solution (dashed lines). The overall agreement between results of the finite-volume method in TD and the finite-element method in FD is very good for all positions and frequencies. The resonances of the two structures are identified at identical frequencies for both methods. A discrepancy is observed in the maximal amplitude of the most resonant modes, which is attributed to the slow convergence of TD simulations for highly resonant structures. The general consistency across two very different numerical methods provides a good confidence that the results are reliable for the electromagnetic modeling of plasmonic nano particles.

Fig. 8 shows the distribution of the field enhancement along the $x$-axis at the observed central wavelength of the resonances. This reveals the influence of the order of the resonance on the enhancement factor and its distribution inside the gap. In the case of an air-filled gap, the resonance at $1335 \mathrm{~nm}$ (Fig. 8 (a), red line) shows maximum enhancement in the edge region, while the resonance at $830 \mathrm{~nm}$ (Fig. 8 (a), green line) has its maximum in the center region. 

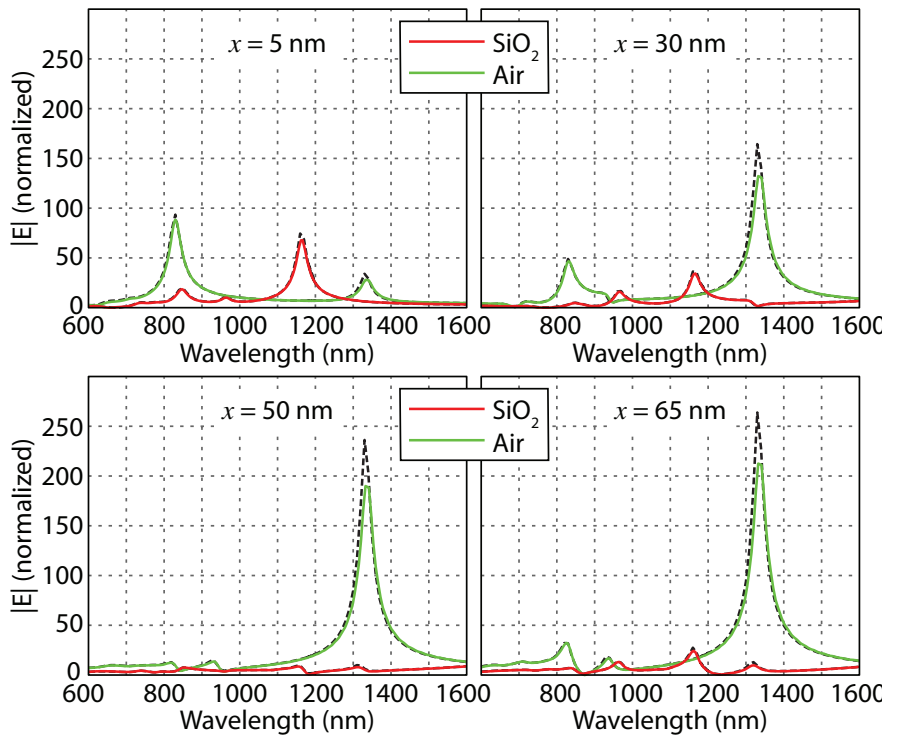

Fig. 7: Simulated field enhancement (FVTD: solid lines, JCMsuite: dashes lines) in the gap filled with air and $\mathrm{SiO}_{2}$.

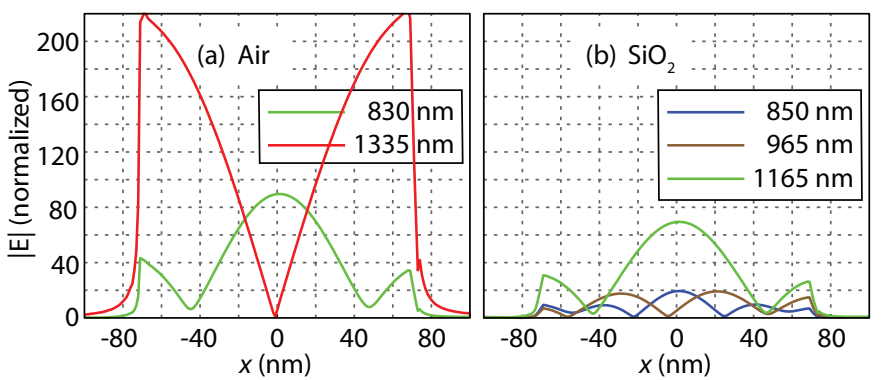

Fig. 8: FVTD simulated field enhancement in the gap filled with (a) air and (b) $\mathrm{SiO}_{2}$ along the $x$-axis at the resonance frequencies.

For the $\mathrm{SiO}_{2}$-filled gap, due to the well-known red shift of plasmonic resonances with increasing refractive index of the dielectric, the mode with maximum enhancement in the center region appears at $1165 \mathrm{~nm}$ (Fig. 8 (b), green line) while the edge-dominated mode is shifted out of the analyzed wavelength range. The observed resonances at $850 \mathrm{~nm}$ and $965 \mathrm{~nm}$ are higher-order modes that show additional maxima and minima along the $x$-axis. As can be observed in Fig. 8, the distribution of the electromagnetic field inside the gap region is not strictly rotationally symmetric (however, it is symmetric with respect to the $x z$ plane). This is due to the plane wave excitation, which is not rotationally symmetric.

Simulations like these can greatly contribute to the successful design of such plasmonic structures and can be extended to more complex devices with a specific functionality. This is particularly relevant as the nano-fabrication processes are costly and time consuming. It is well known that the central wavelength and the magnitude of the resonances of coupled plasmonic systems depend strongly on gaps, on the material and on the dimensions of the metallic nanostructures [12]. Additionally, as shown here, the field strength at a resonant wavelength varies strongly within the region of interest, showing characteristic mode-dependent distributions. In the illustrated case, either the fundamental or a higher-order mode is favorable, depending on the targeted application. The firstorder mode is to be preferred if high enhancement factors in the edge region are needed. One the other hand, higher-order modes are favorable for applications based on the center region of the gap. Additionally, it can be expected that these modes are less influenced by manufacture tolerances and ageing.

\section{CONCLUSIONS}

The plasmonic resonances of stacked nanodisks were numerically investigated in this paper. The simulated field enhancement obtained by JCMsuite and FVTD showed good agreement over the whole wavelength spectrum of interest, although both methods are based on different concepts and are applied in frequency and in time domain, respectively. The direct observation of the modes inside the plasmonic gap is an important step in the development process. This can be conveniently achieved by electromagnetic numerical modeling, providing a physical understanding essential for a successful design.

\section{References}

[1] M. Danckwerts and L. Novotny, "Optical Frequency Mixing at Coupled Gold Nanoparticles," Phys. Rev. Lett. vol. 98, pp. 1-4, 2007.

[2] D.J. Maxwell, J.R. Taylor, S. Nie, "Self-Assembled Nanoparticle Probes for Recognition and Detection of Biomolecules," J. Am. Chem. Soc., vol. 124, no. 32, pp. 9606-9612, 2002.

[3] Z. Liu et. al., "Plasmonic Nanoantenna Arrays for the Visible," Metamaterials, vol. 2, pp. 45-51, 2008.

[4] K. Kneipp, Y. Wang, H. Kneipp, L.T. Perelman, I. Itzkan, R.R. Dasari, M.S. Feld, "Single Molecule Detection Using Surface-Enhanced Raman Scattering (SERS)," Phys. Rev. Lett., vol. 78, pp. 1667-1670, 1997.

[5] Y. Ekinci, A. Christ, M. Agio, O.J.F. Martin, H.H. Solak, J.F. Löffler, "Electric and magnetic resonances in arrays of coupled gold nanoparticle in-tandem pairs," Optics Express, vol. 16, no. 17, pp. 13287-13295, 2008.

[6] P. Bonnet, X. Ferrieres, B.L. Michielsen, P. Klotz, J.L. Roumiguieres, "Time domain electromagnetics," chapter 9, pp. 307-367, Academic Press, 1999.

[7] R.J. Gehr, R.W. Boyd, "Optical Properties of Nanostructured Optical Materials," Chem. Mater., no. 8, pp. 1807-1819, 1996.

[8] C. Fumeaux, D. Baumann, P. Leuchtmann, R. Vahldieck, "A generalized local time-step scheme for efficient FVTD simulations in strongly inhomogeneous meshes," IEEE Trans. MTT, vol. 52, no. 3, pp.1067-1076, 2004.

[9] http://www.jcmwave.com/

[10] D. Baumann, C. Fumeaux, P. Leuchtmann, R. Vahldieck, "Finite-volume time- domain (FVTD) modeling of a broadband double-ridged horn antenna," Int. J. Numer. Model., vol. 17, pp.285-298, 2004.

[11] D. Baumann, C. Fumeaux, C. Hafner, E.P. Li, “A Modular Implementation of Dispersive Materials for Time-Domain Simulations with Application to Gold Nanospheres at Optical Frequencies," Optics Express, vol. 17, no. 17, pp. 15186-15200, 2009.

[12] S.A. Maier, "Plasmonics, Fundamentals and Applications," Springer, 2007. 